\title{
COMERCIALIZAÇÃO E PRODUÇÃO DE FOLHAGEM ORNAMENTAL DE CORTE NO ESTADO DE SÃO PAULO
}

\author{
LÚCIA OSHIRO ${ }^{1}$; TAÍS TOSTES GRAZIANO² e MARIA ESMERALDA S. P. DEMATTÊ
}

\section{RESUMO}

Nos últimos anos, as folhagens de corte têm apresentado um crescimento visível na sua produção, tanto do ponto de vista qualitativo quanto quantitativo. Interessado em conhecer um pouco mais a atividade, o trabalho buscou obter informações da produção e da comercialização desses produtos no Estado de São Paulo, a partir de levantamento junto a dois grandes mercados paulistas: CEASACampinas e CEAGESP. Observou-se a existência de 37 pontos de venda desses produtos, sendo nove especializados na atividade, e cerca de 90 produtos disponíveis, sem padronização oficial. Abastecem esses mercados 36 produtores, dos quais apenas oito, especializados. A atividade aparece como complementar à produção de flores e/ou de folhagens envasadas, normalmente em pequenas áreas marginais, sem uso de estruturas e técnicas de cultivo mais especializadas.

Palavras-chave: planta verde de corte, floricultura.

\section{ABSTRACT \\ Commercialization and production of cut ornamental foliage in the State of São Paulo, Brazil}

The cut ornamental foliage has shown a large increasing in production in the last years, quantitative and qualitative. The research seek for more in- formation about production and commercialization of cut foliage in the State of São Paulo. The main interest is the two large markets in the State, CEAGESP and CEASA-Campinas. The research point 37 market points, 9 specialized in the activity, and around 90 products available in the market. Those markets are feed by 36 producers and 8 specialized only. The production of cut ornamental foliage is a activity complements in most case the flower and potted plants production, usually in side areas with no structure nor specialized cultivate techniques.

Key-words: foliage plants, floriculture.

\section{INTRODUÇÃO}

O setor de floricultura vem-se alterando, nos últimos anos, com o crescimento, a especialização e a diversificação da produção, consolidando-se como uma atividade de importância socioeconômica, principalmente para o Estado de São Paulo.

Pela multiplicidade de microclimas dentro do Estado e de técnicas culturais utilizadas, a produção de flores e plantas ornamentais é bastante diversificada, disponibilizando uma variabilidade de produtos que vão desde flores e folhagens para corte e vaso, mudas de ornamentais diversas e material propagativo, como bulbos, cormos, estacas e rizomas, entre outros. Dados obtidos em 1995 (ARRUDA et al., 1996) estimavam que a produção paulista de flores e folhagens de corte ocupava uma área de 436 ha; destes, 175 ha sob estufa, onde prevaleciam

\footnotetext{
${ }^{1}$ Engenheira Agrônoma, estágio curricular do Curso de Agronomia da FCAV-UNESP, junto ao Centro de Análise e Pesquisa Tecnológica do Agronegócio da Horticultura - IAC.

${ }^{2}$ Instituto Agronômico - Centro de Análise e Pesquisa Tecnológica do Agronegócio da Horticultura - IAC, Caixa Postal 28, 13001-970 Campinas (SP). E-mail: tais@iac.br

${ }^{3}$ Departamento de Produção Vegetal - FCAV-UNESP, Via de Acesso Prof. Paulo Donato Castellane, km 5, 14884-900 Jaboticabal (SP). E-mail: maria@fcav.unesp.br
} 
espécies floríferas como crisântemo, gipsófila, lisianto e gérbera. Apesar de os dados incluírem as folhagens de corte, hoje bastante utilizadas na composição de arranjos florais e, por isso, de extrema importância para os floristas, pouco se sabe sobre a sua produção, tanto em termos qualitativos quanto quantitativos.

Como folhagens ou planta verde de corte, é considerada uma série de produtos, necessariamente folhas. Além delas propriamente ditas (marantáceas, palmeiras e aráceas), entram ramos enfolhados, herbáceos (aspargos, samambaias, heras) e lenhosos (tuias, pitósporo, eucalipto), ramos abotados (vime-azuki) ou simplesmente nus (Costus stenophyllus), enfim, qualquer porção da parte aérea de uma planta sem flor, com característica ornamental que possa compor arranjos florais.

O cultivo de folhagens para corte não é recente, porém poucos eram os produtos ofertados até a década dos oitentas. Alguns deles, pela ocorrência natural em abundância em determinadas regiões, eram simplesmente dali extraídos, como a guaricanga (Geonoma pohliana) e o avencão (Rhumora adiantiformis) na Ilha Comprida, outros cultivados em pequena escala, como a samambaia-paulistinha (Nephrolepsis pectinata (Willd.) Schott), os aspargos ornamentais (Asparagus spp.), o eucalipto-cinzento (Eucaliptus cineraea F. Muell), a monstera (Monstera deliciosa Liebn.) e as dracenas (Dracaena spp.).

Segundo LAMAS (2002), os arpargos ornamentais são as folhagens de corte de emprego mais tradicional, sendo cultivados desde o final do século XIX. A partir de meados do século XX, no entanto, é que se deu certo impulso na produção de folhagens com a introdução do cultivo do avencão e a adoção de novas técnicas. Mesmo assim, há pouco mais de cinco anos, a produção paulista de aspargos ornamentais de corte não representava mais que seis mil pés em cultivo, sendo um hectare a céu aberto e $3.000 \mathrm{~m}^{2}$ em estufa, entre dois produtores entrevistados, com produção anual de 12.400 maços (ARRUDA et al., 1996). Atualmente, maior variedade desse tipo de produto vem sendo comercializada, em diferentes formas, tamanho e coloração, a qual está conquistando mercado e ganhando, com isso, uma importância antes secundária no setor. Desse modo, têm-se efetuado levantamentos (OSHIRO, 2000 e BROEK, 2000), no sentido de obter dados da produção, da comercialização, da padronização e do desenvolvimento de mercado com a finalidade de diagnosticar a atividade, apontando as oportunidades e os gargalos, de forma a orientar as atividades e as políticas a adotar, em todos os elos da cadeia produtiva.

\section{A COMERCIALIZAÇÃO DE FOLHAGEM CORTADA}

A comercialização de folhagens de corte, nos três principais mercados do Estado de São Paulo (CEAGESP, CEASA-Campinas e Veiling-Holambra), é feita quase que exclusivamente do produtor para grandes distribuidores de plantas (atacadistas), que as entregam para os floristas (no varejo) e empresas de decoração.

Em levantamento junto ao Mercado Permanente de Flores da CEASA, identificaram-se 14 pontos de comercialização de folhagem de corte, sendo um atacadista e os outros, produtores. Destes, apenas quatro são especializados na atividade, tendo os demais a comercialização de folhagens como um complemento da venda de flores cortadas. Já no mercado de flores da CEAGESP, que funciona às terças e sextas-feiras, de manhã, foram levantados 23 comerciantes, todos também produtores. Desses, apenas cinco tinham na folhagem de corte sua atividade principal (OSHIRO, 2000).

Junto aos boxes de comercialização, no período de agosto a novembro, identificaram-se 64 espécies de folhagens cortadas, que se encontram na Tabela 1 pelos nomes popular e científico, número de produtores identificados e o local de produção.

No período julho-setembro de 2000, BROEK (2000) identificou 23 outros produtos, entre a CEASA, a CEAGESP e o Veiling, a saber: Ammi majus, ameixa (Prunus serrulata), araucária (Araucaria heterophylla), Asclepsias physocarpa, Asparagus falcatus, azálea (Azalea sp.), cala-tigrada (Zanthedeschia aethiopica), palmeira-cariota (Caryota mitis), cotinus (Cotinus cogginera), cróton (Codiaeum variegatum), espada-de-são-jorge (Sanseviera trifasciata), flor-decera (Hoya carnosa), Stephanotis floribunda, helicônias (Heliconia spp.), marantas (Marantha spp.), hera-de-inverno (Parthenocissus tricuspidata), hera-roxa (Hemigraphis colorata), monstera-do-amazonas (Monstera adansonii), palmeira-leque (Chamaeropsis humilis), pândano-amarelo (Pandanus baptisti), protea (Protea sp.), russélia (Russelia equisetiformis), estrelítzia (Strelitzia juncea) e guaimbê (Philodendron sellum). 
Tabela 1. Espécies comercializadas na CEASA-Campinas e na CEAGESP, de agosto a novembro de 2000 (OSHIRO, 2000)

\begin{tabular}{|c|c|c|c|}
\hline Nome popular & Nome científico & $\begin{array}{c}\mathrm{N} .^{\circ} \text { de } \\
\text { produtores }\end{array}$ & Municípios produtores \\
\hline 1. Acácia-mimosa .................. & Acacia podalyriaefolia & 1 & Atibaia \\
\hline 2. Âmi ... & Ami majus & 2 & Ibiúna \\
\hline 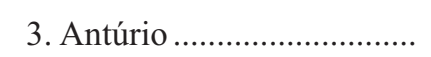 & Anthurium andraeanum & 1 & Registro \\
\hline 4. Areca-bambu ...................... & Dypsis lutescens & 7 & Registro, Cabreúva e Ibiúna \\
\hline 5. Aspargo-alfinete ............... & Asparagus densiflorus var. sprengeri & 6 & Jundiaí, Ibiúna, Cabreúva e Atibaia \\
\hline 6. Aspargo-melindre ............ & A. setaceus & 7 & Jundiaí, Ibiúna e Atibaia \\
\hline 7. Aspargo-micro ...................... & A. myriocladus & 5 & Jundiaí, Ibiúna e Jacareí \\
\hline 8. Aspargo-real ....................... & A. densiflorus cv. myersii & 5 & Atibaia, Jundiaí e Ibiúna \\
\hline 9. Aspargo-smilax .................. & A. asparagoides var. myrtifolius & 7 & Ibiúna \\
\hline 10. Aspargo-vassoura ............. & A. densiflorus cv. pyramidalis & 5 & Atibaia, Jundiaí e Ibiúna \\
\hline 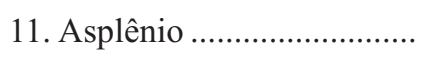 & Asplenium midus & 1 & Atibaia \\
\hline 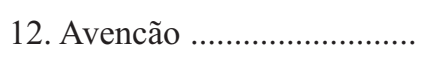 & Rumohra adiantiformis & - & Extrativismo \\
\hline 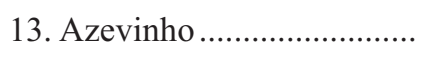 & Ilex aquifolium & 2 & Ibiúna \\
\hline 14. Bambu-de-jardim ............. & Bambusa gracilis & 1 & Atibaia \\
\hline 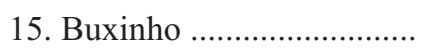 & Buxus sempervirens & 1 & Atibaia \\
\hline 16. Café-de-salão ..................... & Aglaonema communatum & 1 & Registro \\
\hline 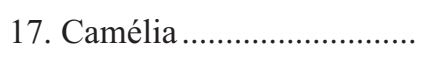 & Camellia japonica & 3 & Ibiúna e Atibaia \\
\hline 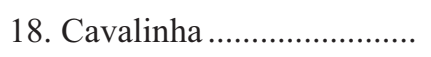 & Equisetum giganteum & 6 & Atibaia, Ibiúna e Jundiaí \\
\hline 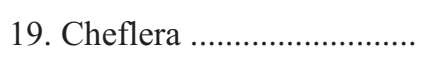 & Schefflera arboricola & 6 & Jundiaí, Campinas, Atibaia e Ibiúna \\
\hline 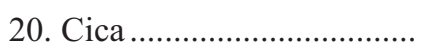 & Cycas revoluta & 1 & Atibaia \\
\hline 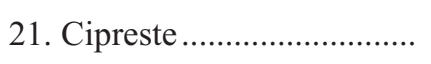 & Cupressus spp. & 4 & Atibaia, Jundiaí, Cabreúva e Ibiúna \\
\hline 22. Cordiline-vermelha ......... & Cordelyne terminalis & 5 & Registro e Ibiúna \\
\hline 23. Dracena-sanderiana ......... & Dracaena sanderiana & 5 & Registro e Ibiúna \\
\hline 24. Dracena-tricolor ................... & Dracaena marginata & 5 & Registro e Ibiúna \\
\hline 25. Estrelítzia ............................. & Strelitzia reginae & 1 & Itupeva \\
\hline 26. Eucalipto-cinzento ........... & Eucaliptus cinerea & 3 & Jacareí, Atibaia e Jundiaí \\
\hline 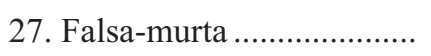 & Murraya exotica & 3 & Peruíbe, Atibaia e Campinas \\
\hline 28. Falsa-seringueira ............. & Ficus elastica var. decora & 1 & Atibaia \\
\hline \multicolumn{4}{|l|}{ 29. Falsa-seringueira } \\
\hline 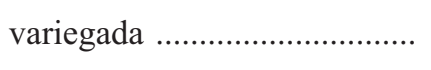 & Ficus elastica var. variegata & 1 & Atibaia \\
\hline 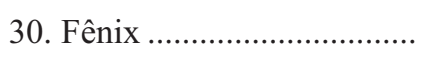 & Phoenix roebelinii & 2 & Atibaia e Registro \\
\hline 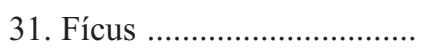 & Ficus benjamina & 1 & Atibaia \\
\hline
\end{tabular}


Tabela 1. Conclusão

\begin{tabular}{|c|c|c|c|}
\hline Nome popular & Nome científico & $\begin{array}{l}\mathrm{N} .{ }^{\circ} \text { de } \\
\text { odutores }\end{array}$ & Municípios produtores \\
\hline 32. Flor-seca-de-lótus ............ & Nelumbo nucifera & 1 & Ibiúna \\
\hline \multicolumn{4}{|l|}{ 33. Fórmio-verde e } \\
\hline 34. Fórmio-variegado ............ & Phormium tenax & - & Várias regiões \\
\hline 35. Guaricanga ........................ & Geonoma pohliana & - & Extrativismo \\
\hline 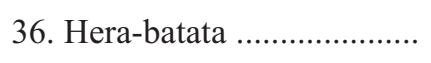 & Hedera canariensis & 5 & Atibaia, Jundiaí, Ibiúna e Itupeva \\
\hline 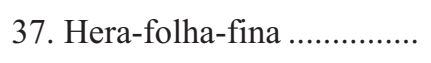 & Hedera helix & 2 & Santo André e Ibiúna \\
\hline 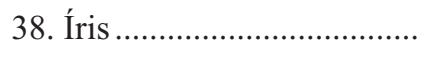 & Iris germanica & 1 & Jundiaí \\
\hline 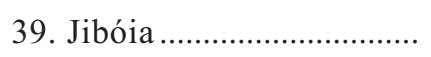 & Scindapsus aureus & 3 & Registro \\
\hline 40. Junco ……......................... & Juncus effusus & 1 & Ibiúna \\
\hline 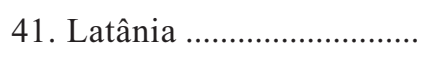 & Livistona chinensis & 5 & Ibiúna, Atibaia e Campinas \\
\hline 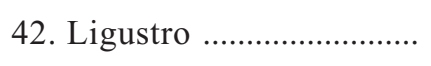 & Ligustrum sinense & 3 & Ibiúna e Atibaia \\
\hline 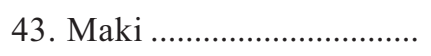 & Podocarpus macrophyllus var. maki & 2 & Jundiaí e Atibaia \\
\hline 44. Minieucalipto ...................... & - & 1 & Atibaia \\
\hline 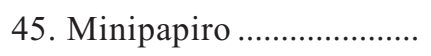 & Cyperus haspan viviparus & 1 & Atibaia \\
\hline 46. Molucela ............................ & Molucella laevis & 2 & Ibiúna \\
\hline 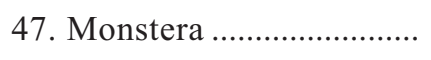 & Monstera deliciosa & 10 & Jundiaí, Atibaia, Registro, Cabreúva \\
\hline 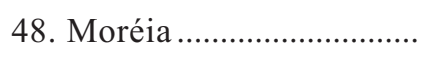 & Dietes bicolor ou Dietes iridioides & 1 & Atibaia \\
\hline 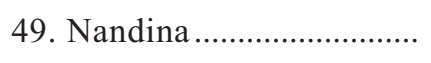 & Nandina domestica & 1 & Atibaia \\
\hline 50. Papiro-chinês ................... & Cyperus alternifolius & 1 & Atibaia \\
\hline 51. Papiro-do-egito ................ & Cyperus papirus & 7 & Atibaia, Jundiaí e Cabreúva \\
\hline 52. Pinheiro-matsu ................ & Pinus parviflora & 1 & Atibaia \\
\hline 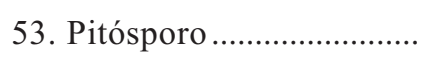 & Pittosporum tobira & 5 & Holambra, Atibaia e Cabreúva \\
\hline 54. Renda-portuguesa .......... & Davallia fejeensis & 1 & Registro \\
\hline 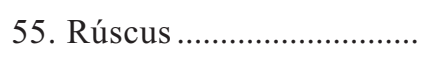 & Ruscus sp. & 5 & Ibiúna e Atibaia \\
\hline 56. Samambaia-paulistinha & Nephrolepis pectinata & 3 & Guararema, Registro e Atibaia \\
\hline 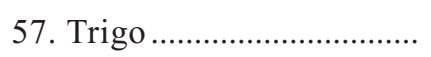 & Triticum aestivum & 3 & Atibaia \\
\hline 58. Tuia-dourada ………............ & Chamaecyparis obtusa var. crippssii & 3 & Ibiúna e Atibaia \\
\hline 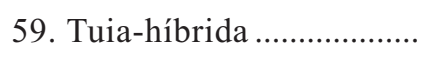 & Thuja orientalis var. rosedalis-aurea & 3 & Ibiúna e Atibaia \\
\hline 60. Tuia-prateada ..................... & Chamaecyparis lawsoniana var. albo-picta & ta 3 & Ibiúna e Atibaia \\
\hline 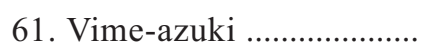 & Salix sp. & 5 & Atibaia \\
\hline 62. Vime-bola .......................... & Salix sp. & 5 & Atibaia \\
\hline 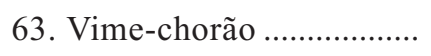 & Salix sp. & 5 & Atibaia \\
\hline 64. Vime-dragão ..................... & Salix sp. & 5 & Atibaia \\
\hline 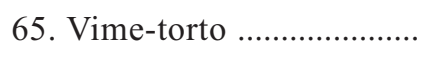 & Salix sp. & 5 & Atibaia \\
\hline
\end{tabular}


As diferenças encontradas nos levantamentos realizados por OSHIRO (2000) e BROEK (2000) indicam que a oferta dos produtos pode variar em função da época do ano, mostrando uma sazonalidade na produção, e do mercado e/ou região produtora. Não dá para auferir que a presença desses novos produtos seja apenas em função do acréscimo do Veiling na amostra como mercado, mas, provavelmente, também pela diferença entre os meses levantados.

Pelo seu cultivo recente e talvez até pela variedade de produtos, não existe uma padronização oficial para folhagens de corte, sendo comercializadas sem preocupação com a classificação e com a embalagem, tampouco com tratamentos de pós-colheita, o que, de certa maneira, compromete a qualidade dos produtos. Não obstante, observa-se certo padrão, quanto à quantidade de hastes por unidade comercializada, adotado pela maioria dos comerciantes.

BROEK (2000) enfatizou que a padronização traria ao mercado uma logística de distribuição melhor, assim como melhoraria também a comunicação entre os agentes do setor, tornando-a mais eficiente.

Os produtos mais procurados pelos clientes, de modo geral, talvez por sua maior tradição, são as folhas de avencão (Rumohra adiantiformis), aspargos ornamentais, tuias (Chamaecyparis obtusa var. albopicta, Thuja orientalis var. rosedalis-aurea, Chamaecyparis obtusa var. crippssii), vimes, fórmio, moréia (Dietes bicolor e D. iridioides), folhas de camélia e eucalipto, além do trigo. No mercado de flores do CEAGESP, mais especificamente, acrescentam-se a esses: junco, cipó-de-kiwi, galho de pêra, ligustro, pitósporo, tuias, cheflera e guaricanga - Figura 1.

Apesar de não haver falta de produtos durante o ano, a venda não é constante, tendo picos em datas comemorativas - Dia das Mães, Finados, início da primavera, Ano Novo e Dia dos Namorados demonstrando ser um mercado sazonal, como relatou CASTRO (1998).

Dados obtidos junto ao Veiling-Holambra, referentes à comercialização de folhagens cortadas, em 1999-2000, mostram um aumento de $21 \%$ na quantidade de maços comercializados, acompanhado por uma queda de $8,2 \%$ no preço médio do período Tabela 2.
Tabela 2. Quadro comparativo de vendas de folhagens, com as quantidades e preços médios praticados, em 1999-2000, no Veiling-Holambra, 2000

\begin{tabular}{|c|c|c|c|}
\hline Ano & Quantidade & Preço & Volume \\
\hline & maços & & $\mathrm{R} \$$ \\
\hline 1999 & 30.699 & 1,83 & $56.179,17$ \\
\hline 2000 & 37.139 & 1,68 & $62.393,52$ \\
\hline Aumento $\%$... & 20,98 & $-8,18$ & 11,06 \\
\hline
\end{tabular}

Apesar de o volume comercializado ainda ser pequeno, comparado com o das flores tradicionais, observa-se um crescimento na comercialização, tanto do ponto de vista qualitativo quanto quantitativo.

\section{A PRODUÇÃO}

Os produtos oferecidos nos mercados paulistas vêm, em grande parte, do próprio Estado de São Paulo, principalmente dos municípios de Jundiaí, Cabreúva, Atibaia, Santo André, Ibiúna, Peruíbe e Registro, como, também, dos Estados do Rio de Janeiro, Santa Catarina e Rio Grande do Sul, sendo os dois últimos os maiores fornecedores de guaricanga e de avencão.

Em levantamento junto à CEAGESP e à CEASA em 2000, encontraram-se 36 produtores de folhagens de corte, sendo que somente oito deles se dedicavam exclusivamente ao cultivo desse tipo de produto. A maioria não tinha na produção de plantas verdes de corte a sua atividade principal, aparecendo esta como um complemento da produção de flores de corte ou envasadas. Algumas espécies, como a guaricanga e o avencão, ainda são, na sua maioria, extraídas da mata, enquanto muitas delas são obtidas de áreas marginais da propriedade, onde foram plantadas com outras finalidades (cercas-vivas, quebra-ventos, etc) e acabaram tendo seu valor ornamental descoberto.

As espécies cultivadas de forma mais intensiva representam uma pequena parcela do todo. Cada produtor tem um sistema de produção diferente que, pelo que se pode notar, foi sendo adaptado pela própria experiência, sem adoção de qualquer tecnologia específica. 

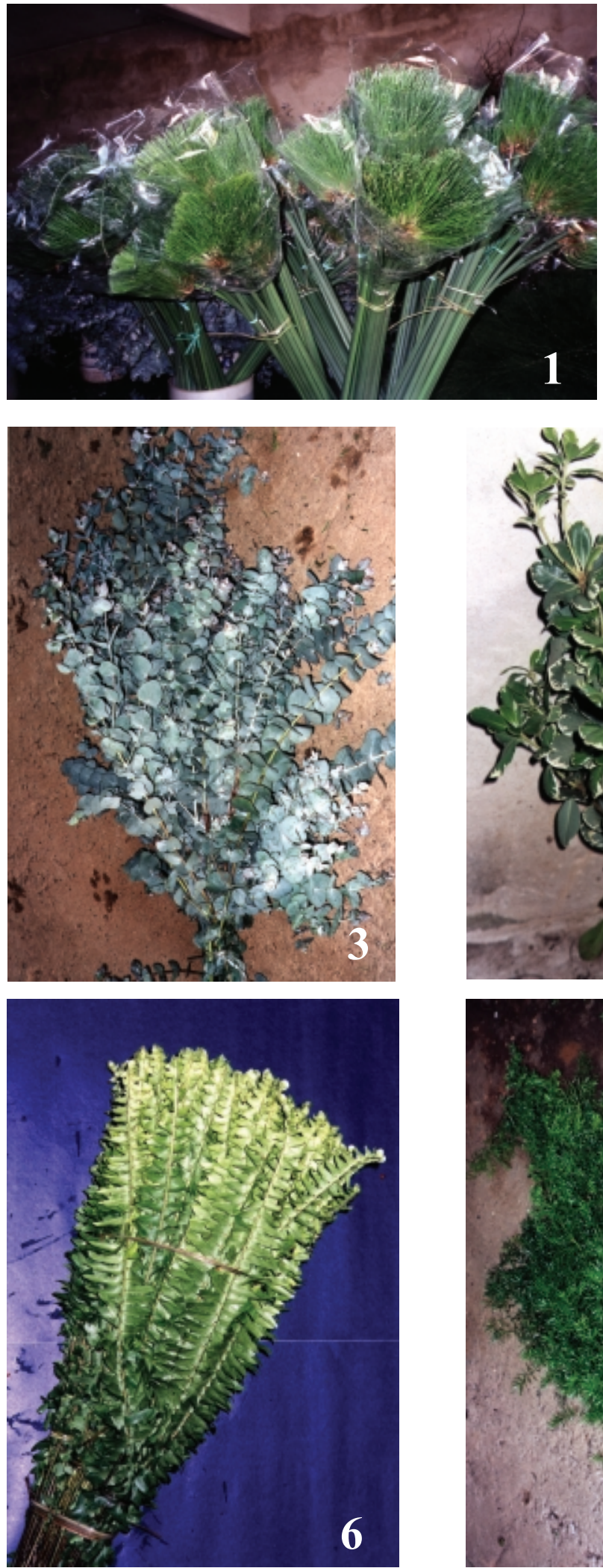
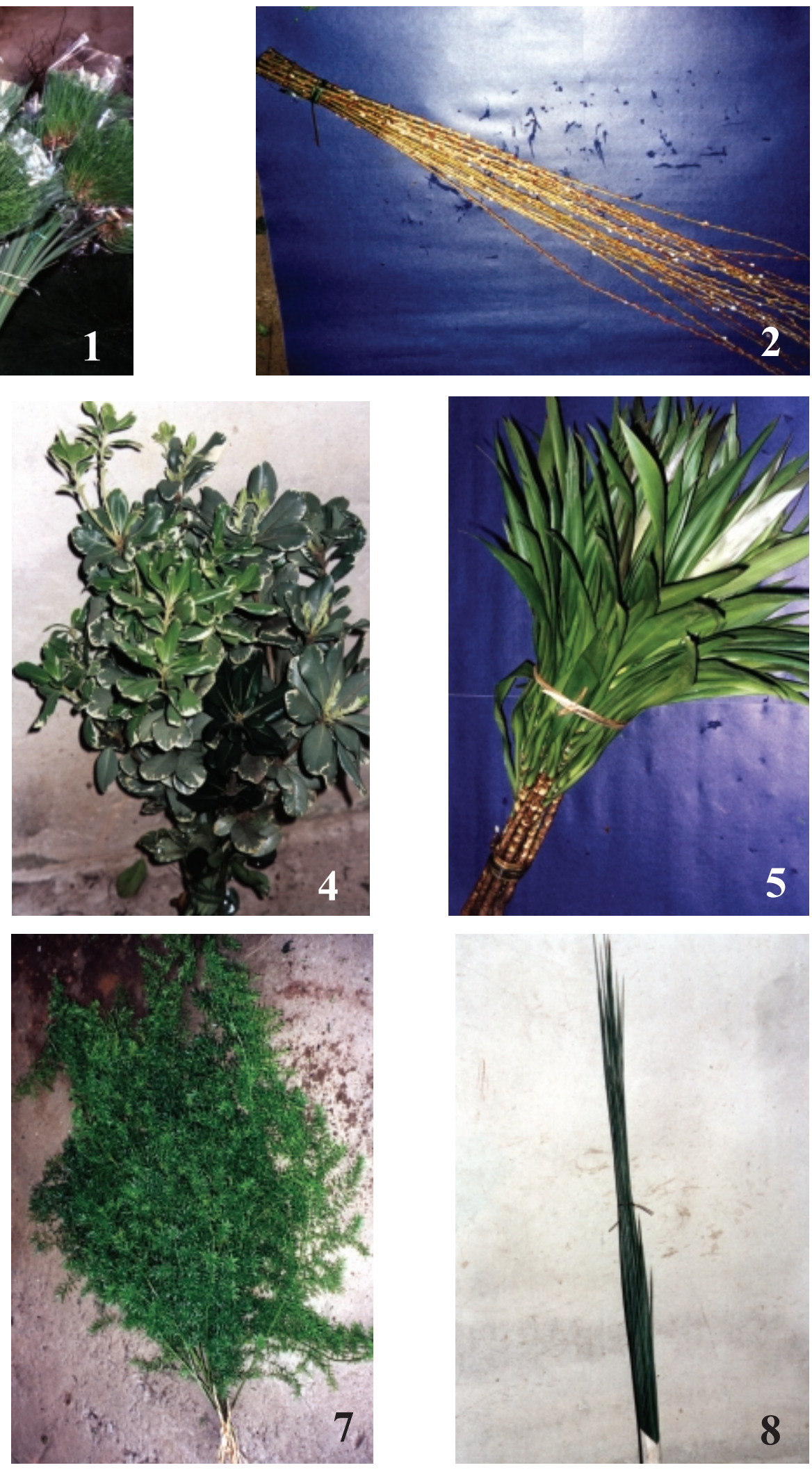

Figura 1. Maços de folhagens de corte comercializados nos mercados tradicionais do Estado de São Paulo. 1: Cyperus papirus; 2: vime-azuki; 3: Eucaliptus cinerea; 4: Pittosporum tobira; 5: Dracaena sanderiana; 6: Nephrolepsis pectinata; 7: Asparagus densiflorus var. sprengeri e 8: Juncus effusus. 

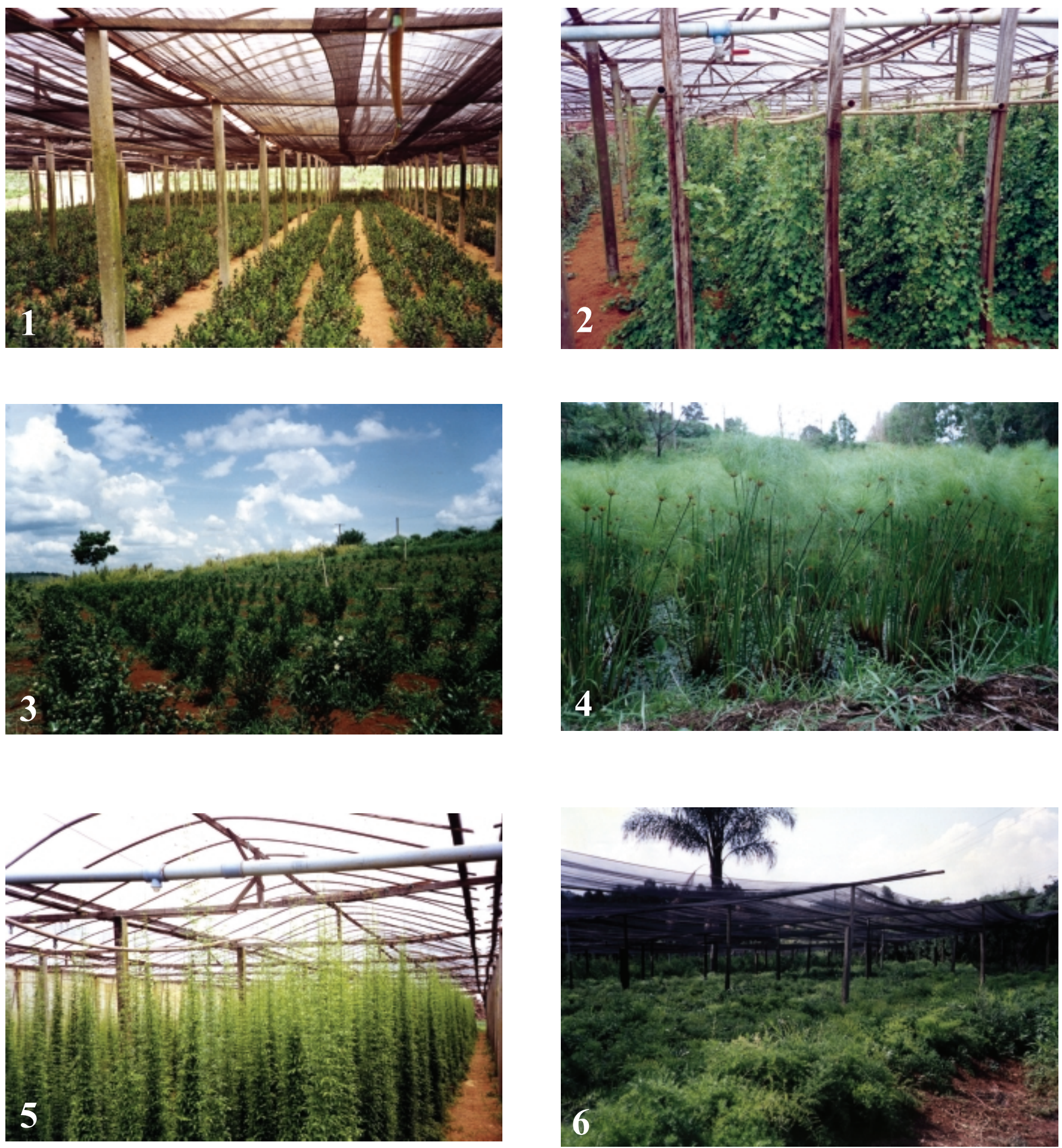

Figura 2. Detalhes da produção de folhagens para corte em campo aberto e sob cultivo protegido. 1: Ruscus sp.; 2: Hedera helix; 3: Camellia japonica; 4: Cyperus papirus; 5: Asparagus asparagoides var. myrtifolius e 6: Asparagus densiflorus var. sprengeri. 
Por se tratar de uma produção secundária, em pequenas áreas e com certa diversidade, não costuma apresentar os problemas fitossanitários que em cultivos mais intensivos ocorrem, com as pragas e as doenças.

A produção ocorre tanto em campo aberto, a pleno sol ou sob vegetação, como também em telados ou sob estufas plásticas, conforme a necessidade do produto. Em campo aberto são cultivadas principalmente tuias, camélias, bambu de jardim, pitosporo, nandina, buxinho, papiros, falsa-murta, palmeira fênix, fórmio e junco. Já plantas como areca-bambu, monstera e samambaia paulistinha, entre outras, são cultivadas à sombra, normalmente produzida por espécies arbóreas. O plantio é feito em linhas ou canteiros, com espaçamentos definidos pelos próprios produtores, sem nenhum tipo de orientação. Como trato cultural, empregam-se apenas capinas, que podem ser manuais ou mediante herbicidas.

O cultivo protegido é o indicado para as espécies mais sensíveis, sendo utilizadas estruturas de madeira, metal ou alvenaria cobertas tanto com plástico transparente como com telas plásticas (sombreamento variado), conforme a cultura. Encontram-se, sob cultivo protegido, espécies como a cavalinha, rúscus, molucela, aspargos e hera. Nessa condição, o plantio é feito normalmente em canteiros, utilizando-se vários tipos de tutores na condução das plantas. A cavalinha e a molucela exigem o uso de redes de condução, levantadas conforme o crescimento das plantas; para os aspargos smilax e melindre utilizam-se fios esticados verticalmente, nos quais a planta vai sendo conduzida, exigindo muita mão-de-obra durante essa etapa, enquanto, para a hera, é suficiente o uso de bambus cruzados, como para o tomateiro. Os tratos culturais são a irrigação e as capinas, quando necessárias - Figura 2.
Comparando-se os dados de ARRUDA et al. (1996) com os obtidos no levantamento de OSHIRO (2000), observa-se um aumento não só no número de produtores, de 2 para 36, como também na diversidade de produtos, mostrando a tendência de crescimento da produção.

Apesar da precariedade da produção observada para folhagens de corte, já existem propriedades se especializando, utilizando áreas maiores para o cultivo e tecnologia mais avançada. No entanto, o que se nota é que, para atingir os níveis de qualidade exigidos pelo mercado, atualmente, muito há de se aperfeiçoar para alcançar um nível tecnológico que dê suporte à produção, assim como à pós-colheita desses produtos.

\section{LITERATURA CITADA}

ARRUDA, S. T.; OLIVETTE, M. P. A. \& CASTRO, C. E. F. Diagnóstico da floricultura no Estado de São Paulo. Rev. Bras. Hortic. Ornam., Campinas, v.2, n.2, p.1-18, 1996.

BROEK, D. J. van. Relatório de estágio curricular optativo realizado junto ao Veiling Holambra. Jaboticabal: FCAV-UNESP, 2000. 18p. Relatório.

CASTRO, C. E. F. Cadeia produtiva de flores e plantas ornamentais. Rev. Bras. Hortic. Ornam., Campinas, v.4, n.1/2, p.1-46, 1998.

LAMAS, A. M. Floricultura tropical: técnicas de cultivo. Recife:SEBRAE/PE, (Série...), 2002. 86p.

OSHIRO, L. Relatório de estágio curricular optativo realizado junto ao Instituto Agronômico de Campinas na área de Floricultura. Jaboticabal:FCAV-UNESP, 2000. 62p. Relatório. 